\title{
Dynamic beam switching by liquid crystal tunable dielectric metasurfaces
}

\author{
Andrei Komar, ${ }^{*, \dagger}$ Ramón Paniagua-Domínguez, ${ }^{*, \dagger}$ Andrey Miroshnichenko, Ye \\ Feng Yu, ${ }^{\ddagger}$ Yuri S. Kivshar, ${ }^{\dagger}$ Arseniy I. Kuznetsov, ${ }^{\ddagger}$ and Dragomir Neshev ${ }^{\dagger}$ \\ $\dagger$ Nonlinear Physics Centre, Australian National University, Canberra ACT 2601, Australia \\ $\ddagger$ Data Storage Institute, $A{ }^{*}$ STAR, 138634 Singapore \\ ISchool of Engineering and Information Technology, University of New South Wales, \\ Canberra ACT 2600, Australia
}

E-mail: andrei.komar@anu.edu.au; ramon-paniag@dsi.a-star.edu.sg

\begin{abstract}
Dynamic steering of laser beams by ultra-thin optical metasurfaces is significant research advance for possible applications in remote ranging and sensing. A unique platform for such important functionalities is offered by dielectric metasurfaces having the highest transmission efficiency. However, the realization of dynamically tunable metasurfaces still remains a challenge. Here we demonstrate experimentally the dynamic switching of beam position by all-dielectric metasurfaces composed of silicon nanodisks infiltrated with liquid crystals. In particular, we show the switching of a laser beam from zero to $12^{\circ}$ angle with an efficiency of $50 \%$ by heating the metasurface to modify the liquid crystal state from nematic to isotropic. Our results open important opportunities for tunable ultra-thin beam steering metadevices.
\end{abstract}




\section{Keywords}

optical metasurface, beam steering, dielectric resonators, liquid crystals

All-dielectric metasurfaces have received a great attention in the past years, ${ }^{1-3}$ and than have been established as a platform for efficient manipulation of optical beams. The advances in the design and fabrication of such dielectric metasurfaces have led to the development of several ultra-thin optical metadevices, including flat lenses, ${ }^{4-7}$ beam converters, ${ }^{8-10}$ deflectors $^{9,11-14}$ and holograms. ${ }^{15-17}$ Composed of periodic or aperiodic latices of dielectric nanoparticles, such metasurfaces exhibit low absorption in the infra-red and visible spectral ranges. Low losses allow nanoparticles to exhibit Mie-type resonances with a higher qualityfactor in comparison to their plasmonic counterparts. Furthermore, Mie-type resonances in dielectric nanoparticles offer two independent families of resonant modes - electric and magnetic. The far-field interference of these two types of resonant modes lead to fundamentally new effects, such as unidirectional scattering, ${ }^{18,19}$ unconventioanal reflection behavior associated with the generalized Brewster effect ${ }^{20}$ and near-unity transmission in the so-called Huygens' regime. ${ }^{9,10,21-23}$ The Huygens' regime of operation of the dielectric metasurfaces enables the combination of near-unity transmission together with a full-range of phase modulation thus being the key to enabling functional dielectric metasurfaces with nearly $100 \%$ efficiency.

Most functional dielectric metasurfaces to date are based on static designs, defined solely through a choice of nanoparticle geometry. However, in many applications, it is crucial to enable dynamic tunability of the device functionality with time. For example the focal distance of a camera lens needs to be changed when taking pictures of objects at different distances; the position of the ranging beam in driver-less vehicles needs to scan different directions. Therefore, implementing dynamic control over the response of the metasurfaces is of a paramount importance for their practical implementation.

The development of tunable metasurface optics was first focused on enabling different mechanisms for metasurface tunability. ${ }^{24}$ Nowadays, the focus is shifting towards the demon- 

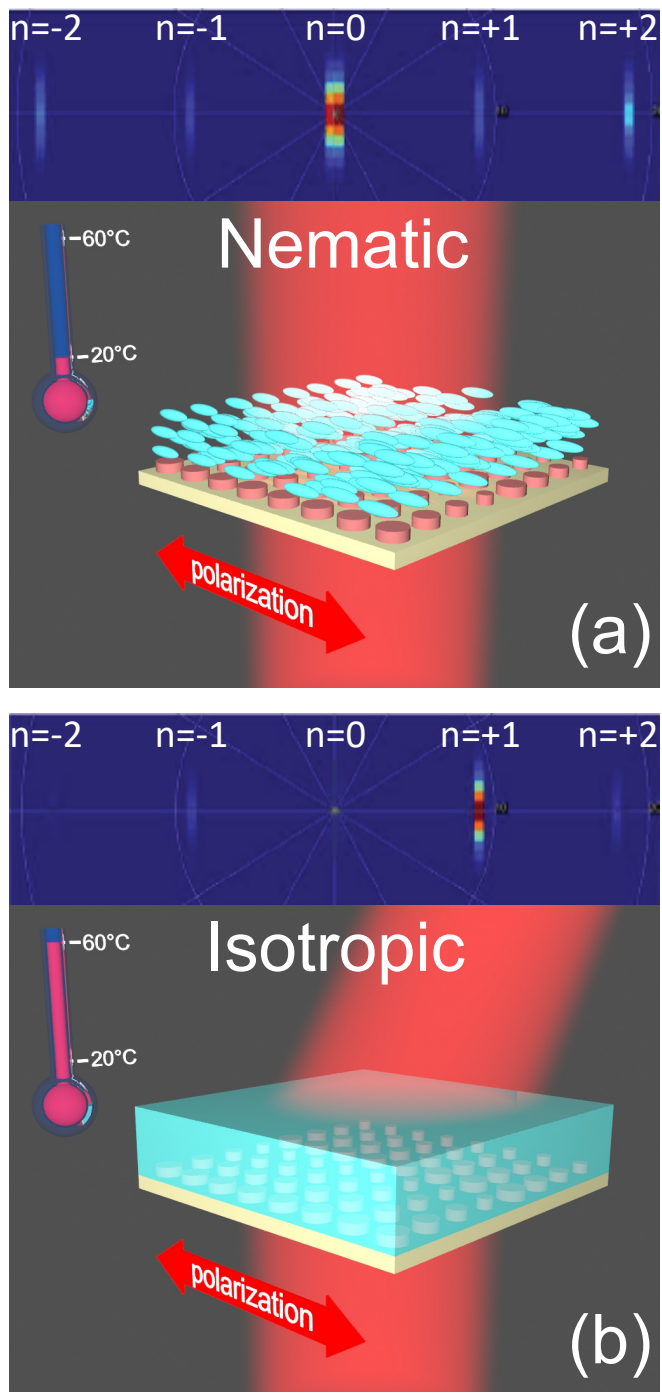

Figure 1: Concept of beam switching metasurfaces infiltrated with liquid crystal. (a) Nematic state. (b) Isotropic state.

stration of tunable functionalities, while high efficiency and possibility to individually control the response of each nanoantenna in a metasurface remain a major challenges. ${ }^{25}$ Several schemes for tunability of resonant dielectric metasurfaces have been proposed enabling mechanical deformation of the structures or a change of the refractive index of either dielectric resonators or surrounding media. These include the mechanical or electrical deformation of elastomers, ${ }^{26,27}$ electrical ${ }^{28}$ or thermal ${ }^{29,30}$ tuning of semiconductors, as well as the use of phase-change materials, ${ }^{31,32}$ graphene, ${ }^{33}$ or liquid crystals (LCs) as embedding media. ${ }^{34-36}$ 
The use of LCs is an attractive approach as it relies on well-developed techniques from the LC display (LCD) industry. LCs possess high optical birefringence that can be controlled by temperature or an electric field. All of these properties make LC-tunable metasurfaces a practical realization of tunable metadevices, which is likely to be favored by the display industry today. To date, however, the LC tunability has only been demonstrated with homogeneous metasurfaces, while tunable functionality of metasurfaces has not been investigated. Here, we experimentally demonstrate a control of the beam steering with the use of gradient all-dielectric metasurfaces infiltrated with LCs. We show that we can effectively switch beam diffraction from the metasurface from the zeroth to the first diffraction order with efficiency of $50 \%$. The tunability is enabled by heating the LC such that it changes its internal structure. At low temperature, the LC is in its nematic phase (Figure 1a), when the elongated LC molecules are arranged parallel to each other. At higher temperatures (Figure 1b) the LC arrangement changes to isotropic. Due to a difference in the refractive indexes between the LC nematic and isotropic states, the metasurface can either transmit light straight through or deflect it at a fixed angle, as shown in Figure 1. In this way, dynamic switching between different beam propagation directions can be achieved.

\section{Results and discussion}

To achieve tuning of an LC-infiltrated metasurface, we first test the properties of a homogeneous nanodisk array. We therefore design and fabricate sub-diffractive silicon-disk arrays operating in the Huygens' regime, e.g. for a spectral overlap of the electric and magnetic dipole resonances of the nanodisks to achieve near-unity transmission. ${ }^{21}$ The array consists of silicon nanodisks with the diameter $d=300 \mathrm{~nm}$, the distance between them $130 \mathrm{~nm}$ and height $h=130 \mathrm{~nm}$, as depicted in Figure 2a. The metasurface was fabricated using an e-beam lithography and subsequent reactive-ion etching. The substrate was quartz with the thickness $0.4 \mathrm{~mm}$. The scanning electron microscopy (SEM) image of this sample is shown 

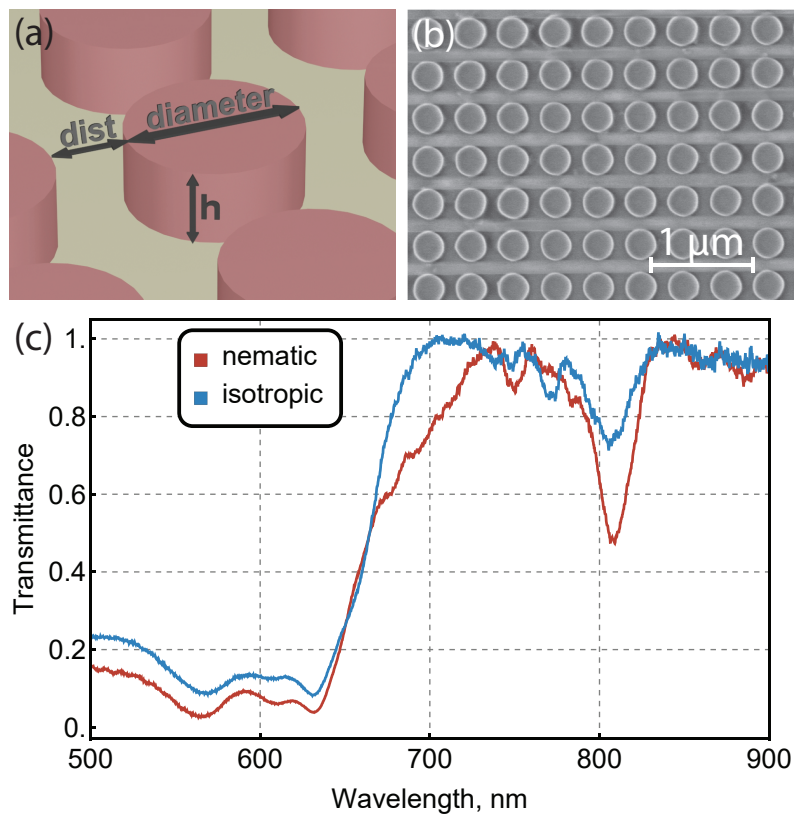

Figure 2: (a) Schematic of a Si nanodisk array metasurface. (b) SEM image of homogeneous metasurface. (c) Transmittance spectrum of the homogeneous metasurface in two liquid crystal states: nematic and isotropic.

in Figure 2b. The exact dimensions were chosen such that the Huygens' regime is satisfied for the isotropic state.

Next, we infiltrate the metasurface with LC to enable the tunability. For our experiments, we chose E7, that is well-investigated and widely used LC in LCD industry. It has positive anisotropy and birefringence $\sim 0.15$ during the transition from nematic to isotropic state for extraordinary wave $n_{e}$ in visible and near infrared wavelength region. ${ }^{37}$ The critical temperature when E7 becomes isotropic is $58^{\circ} \mathrm{C}$. Using this metasurface as a bottom substrate, we build an LC cell with a thickness of $5 \mu \mathrm{m}$ determined by spacers (round plastic balls with precise diameters). We deposit an alignment layer only on the top substrate, while the metasurface remains unaffected. The initial alignment is planar. In optical measurements, we chose the polarization of the incident light along the LC orientation to excite an extraordinary wave inside the cell. For heating the LC cell and controlling its temperature, we use a temperature controller with a control loop, so the measurements for different temperatures are stable. To place the cell with a heating element and temperature sensor in front of the 
microscope objective, we use Mitutoyo objective with high working distance of $2 \mathrm{~cm}$ and numerical aperture $N A=0.7$. To capture the image, we use a CMOS visible camera.

Figure 2c shows the experimentally measured transmittance spectra for both nematic (room temperature) and isotropic (heated sample) alignment of the LC. To reach the LC isotropic state, the LC cell was heated to $60^{\circ} \mathrm{C}$. A change in the transmittance spectrum is observed mostly in the Huygens spectral region at around $810 \mathrm{~nm}$, which is an indication of the resonant behavior of the metasurface. In this region, the transmittance rises from about $45 \%$ to nearly $75 \%$. The observed change in transmittance proves that the alternation of the refractive index of surrounding LC leads to a spectral shift of the resonances. In particular, the magnetic dipolar resonance experiences larger spectral shift ${ }^{34}$ such that it improves the overlap with the electric dipolar resonance in the case of isotropic arrangement of the LC.

After proving the tunability of our Huygens' dielectric metasurface, we proceed to testing the tunability of a functional gradient nanodisk array, such as a beam deflector. ${ }^{9}$ In such a beam deflector, the intensity of the transmitted light is close to unity for the operational wavelength of $800 \mathrm{~nm}$, while its phase is varied such that it forms a binary blazed grating with six phase steps. ${ }^{3,9}$ Each step incorporates the phase delay of $0, \pi / 3,2 \pi / 3, \pi, 4 \pi / 3$ and $5 \pi / 3$, successively. Overall, the device deflects the incident beam at the angle of $12^{\circ}$. It is worth noting that the thickness of the metasurface is only $130 \mathrm{~nm}$, which is much thinner than the operational wavelength in free space. The ultra-small thickness is the main advantage of metasurfaces compared to other diffractive optical elements, which makes them attractive for large-scale nanofabrication.

The schematic of a unite cell of the beam-deflecting metasurface is shown in Figure 3a, and SEM image of the fabricated gradient metasurface is shown in Figure 3b. The height and pitch of the nanodisks are the same as in the homogeneous array described above, however, the diameters of the nanodisks vary such that different phases are imprinted at different spatial locations, while preserving high transmission of the incident beam. This results in a phase gradient that mimics a blazed grating that deflects an incident beam of certain 


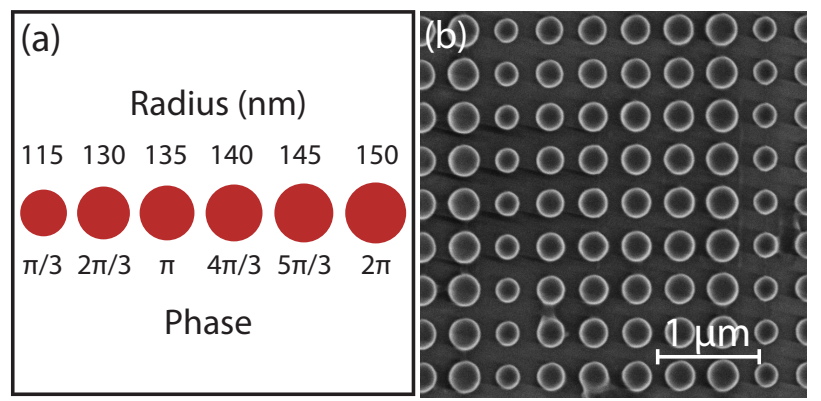

Figure 3: Six-element gradient metasurface. (a) Radii of disks that constitute a unite cell of the beam-deflecting metasurface. (b) SEM image of the fabricated metasurface.

wavelength into the first diffraction order of the grating (see Figure 1).

To determine the exact diameters of the silicon nanodisks which define the phase gradient, first we performed numerical simulations of regular arrays with fixed gaps and heights and varying diameters using Lumerical software. The metasurface is designed to operate when the LC is in the isotropic state and so, the LC is modeled as an isotropic medium. The refractive index used in simulations corresponds to that of the E7 LC above the isotropic phase transition temperature. The results are presented in Supporting information (see Figure S1) and give nanodisk radius varying from $115 \mathrm{~nm}$ to $150 \mathrm{~nm}$ to imprint phase delays in the incoming wave, at the operating wavelength of $780 \mathrm{~nm}$, corresponding to those depicted in Figure 3a.

After defining the required nanodisk diameters, numerical simulations have been done to characterize the performance of the resulting beam-bending metasurface. Results are presented in Figure 4a together with the performance results when the LC is switched to the nematic state, see Figure 4b. In this latter case, the LC is modeled as an anisotropic medium with the extraordinary axis directed along the electric-field polarization direction, which in both cases is perpendicular to the super-cell direction. The values of the extraordinary and ordinary refractive indices correspond to those of the $\mathrm{E} 7 \mathrm{LC}$ at temperature $\mathrm{T}=25^{\circ}$. Panels c-d in Figure 4 show a detailed comparison of the power channeled into the (positive) zero-th, first, and second diffraction orders upon LC switching from the isotropic to nematic state. The results show a power redistribution between the orders, namely, significant reduction of 
(a)

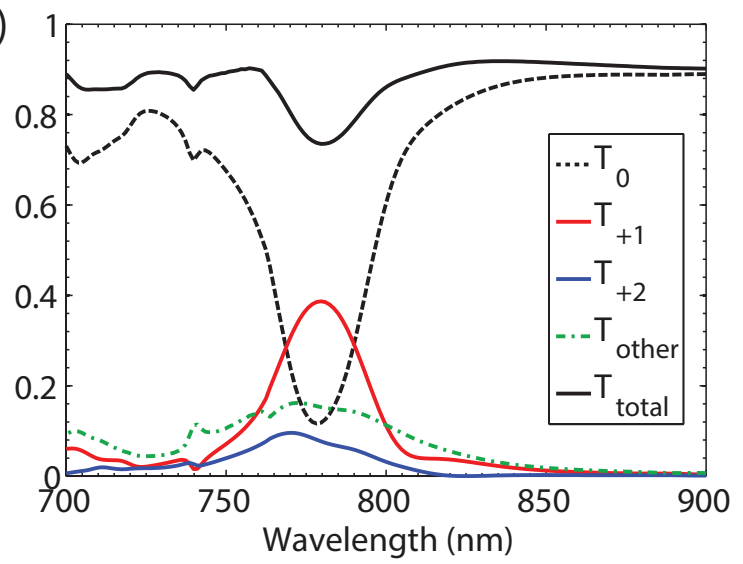

(b)

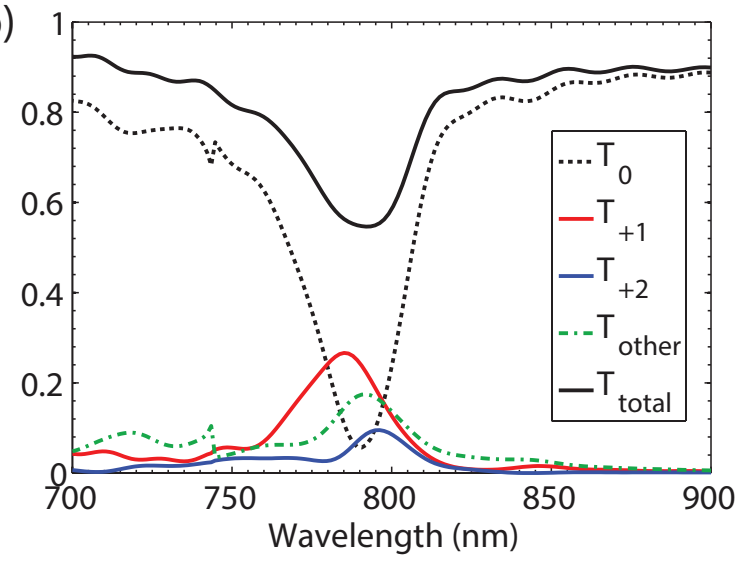

(e)

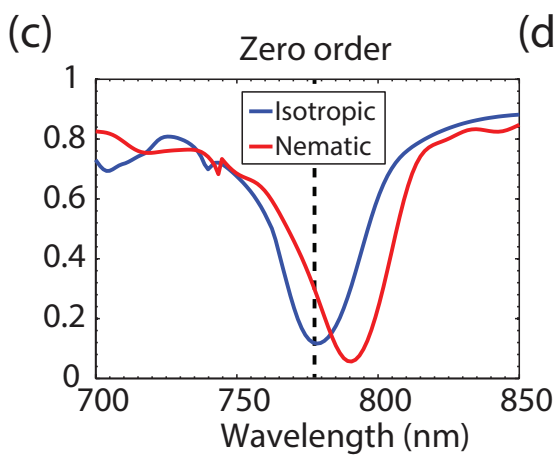

(d)
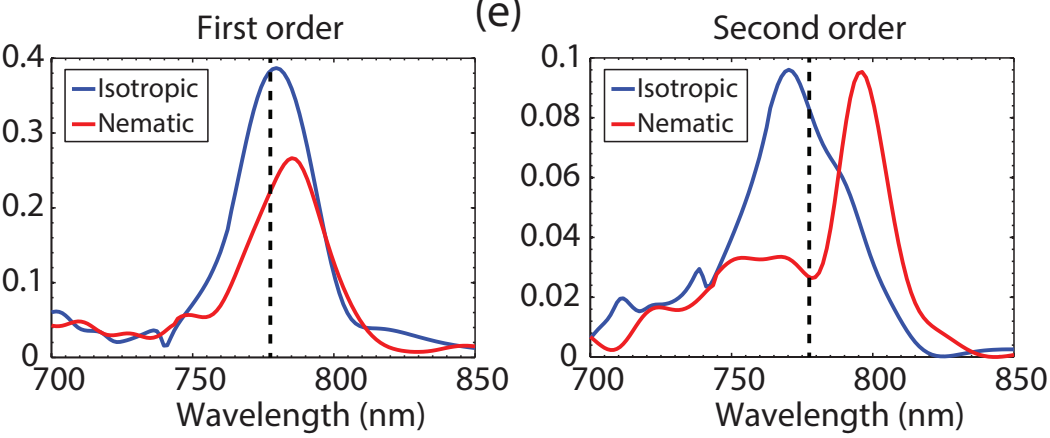

Figure 4: Numerically simulated transmittance spectra for different diffraction orders: (a) Isotropic state of LC, (b) Nematic state of LC. Comparison of zero (c), first (d) and second (e) diffraction orders transmittance for both LC states.

the power channeled into the first order (one enhanced by the gradient metasurface in the isotropic case) and significant increase of the power channeled into the zero-th order (one suppressed by the gradient metasurface in the isotropic case).

To experimentally test the proposed design, the beam-bending gradient metasurface is fabricated, and a typical SEM image of its several unit cells is shown in Figure 3. To perform measurements of the beam deflection, we use an experimental setup shown in Figure S2.

We measure the intensity of each diffraction order for two limiting states of the LC orientation: nematic and isotropic. We implement the measurements of $-2,-1,0,+1,+2$ diffraction orders, however the intensity of -2 and -1 orders is found to be insignificant in both nematic and isotropic states as compared with other orders; therefore in all our figures we demonstrate only $0,+1$, and +2 orders. Based on the numerical simulations (Figure 4 ) we can estimate the range of wavelength operation. However, the fabricated sample has always 
some minor variations in the dimensions compared to the design. Furthermore, since we use a femtosecond laser with $4.4 \mathrm{~nm}$ spectral bandwidth, it is important to define the operational wavelength range directly from the experiment. We therefore measure the intensity of the zero, first and second diffraction orders for different wavelengths. The data for the nematic state are obtained at room temperature $\left(25^{\circ} \mathrm{C}\right)$, and for the isotropic state - at $60^{\circ} \mathrm{C}$. The results are presented in Figure 5 for nematic (red curves) and isotropic (blue curves) states, respectively. The observed increase of the light transmitted into the zero-th order in the nematic case, at around $745 \mathrm{~nm}$, is clearly visible. At the same time, the energy going into the first and the higher diffraction orders is decreased. In this way, we directly obtain the working wavelengths of our beam deflector, which is slightly blue-shifted compared to the initial design.

To demonstrate the switchable properties of our beam-deflection metadevice, we fix the laser wavelength to $\lambda=745 \mathrm{~nm}$ and measure the intensity change of different diffraction orders by increasing temperature of the LC cell. The intensities of different diffraction orders are shown in Figures 6a and 6b. We observe that intensity of the zero-th order decreases with the growth of temperature, while the intensity of the first order increases, the second order remains almost unchanged. All other diffraction orders are not shown in the figures because they carry negligibly low energy as compared to the second order at all measured temperatures. Ideally, a beam deflection metadevice should not only switch the power between $\mathrm{ON}$ and OFF states, but also it should not reduce the total transmitted power. The total transmitted power (a sum of the intensities in all diffraction orders) is given in Figure 6c. During the heating process, the total power decreases only by 20\%, that shows a high transmission efficiency of the device.

To quantitatively show the quality of our beam deflection device, we define its figure of merit. First, a contrast between the intensities of the zeroth and first orders in $\mathrm{ON}$ and $\mathrm{OFF}$ 

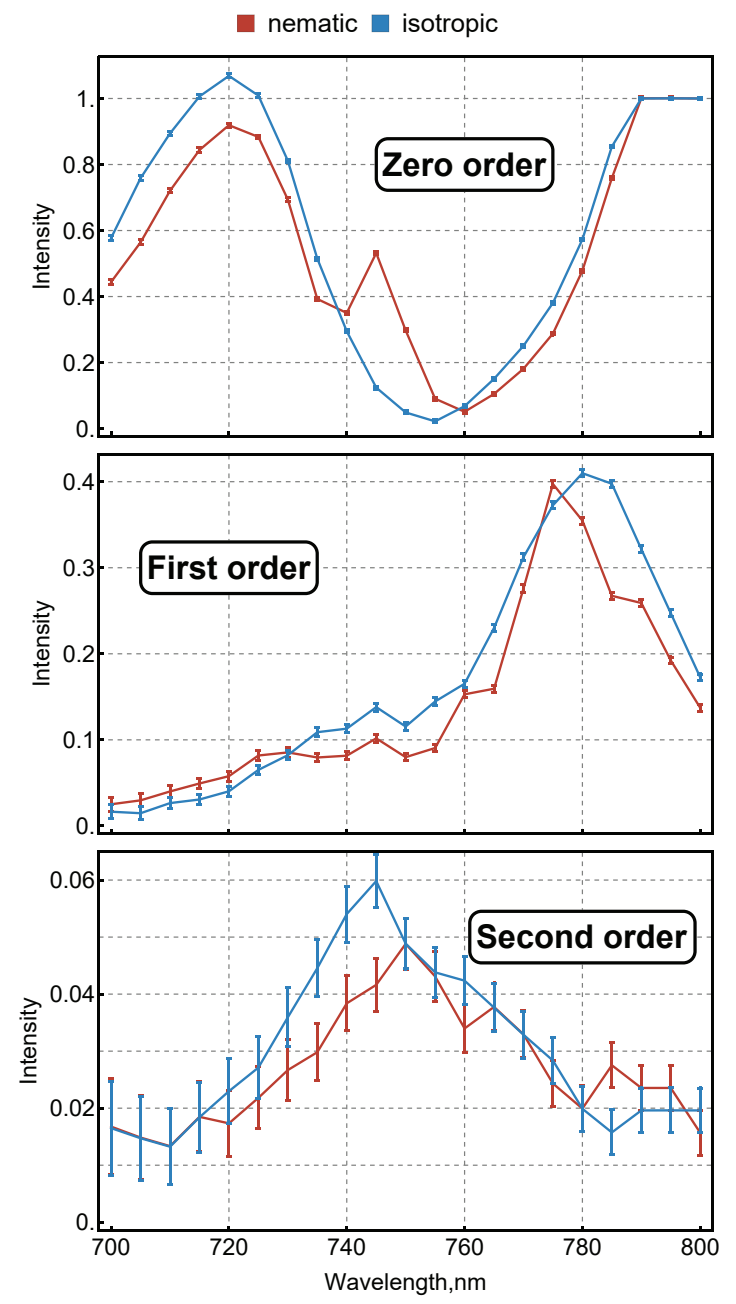

Figure 5: Experimental measurements of the transmittance spectra into the zero-th, first and second diffraction orders in the nematic (red) and isotropic (blue) states of the LC cell. The error bars reflect the level of noise in the measurements.

states of the deflector can be represented as:

$$
C=\frac{I_{0}-I_{1}}{I_{0}+I_{1}}
$$

Here, $I_{0}$ and $I_{1}$ are the intensities of the zeroth and first orders, respectively. This value is between 0 and 1, if $I_{0}>I_{1}$. We define the figure-of-merit $(F O M)$ as a half of the difference between the contrast in $\mathrm{ON}$ and $\mathrm{OFF}$ states: 
(a)

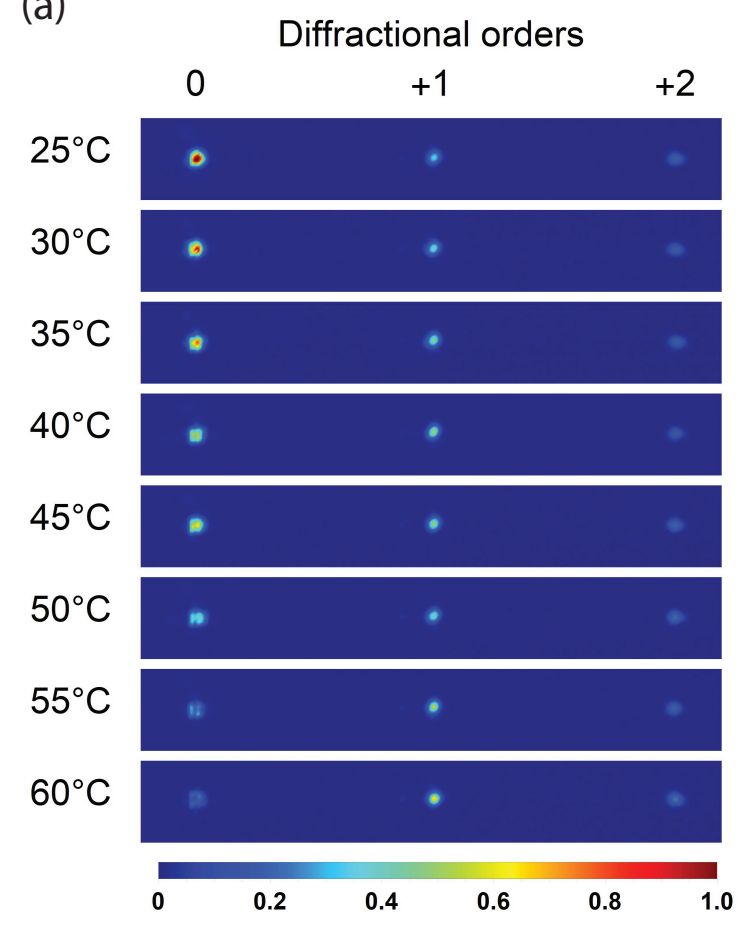

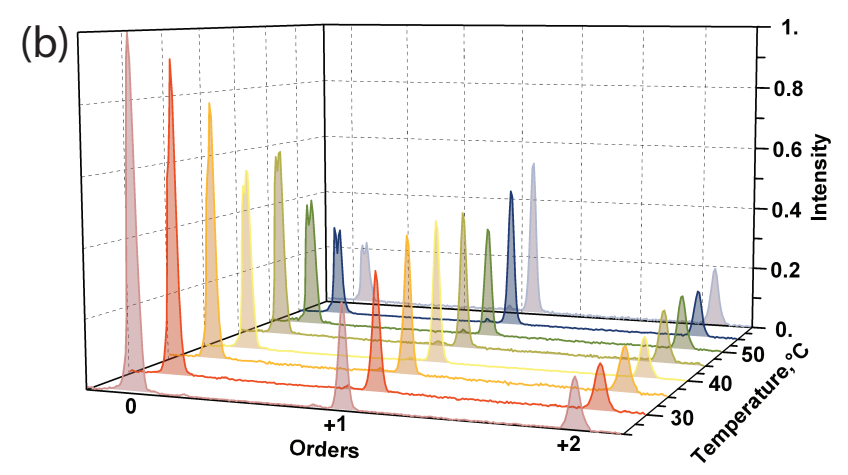

(c)

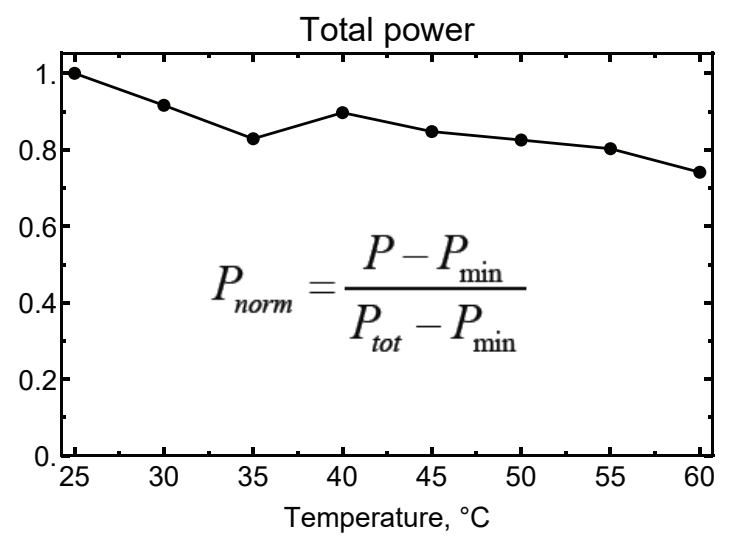

Figure 6: (a) 2D images from CMOS camera for all measured temperatures. (b) The intensity of $0,+1$ and +2 diffraction orders for different temperature of the LC cell. (c) Experimentally measured change of the total power of transmitted light with temperature.

$$
F O M=\frac{1}{2}\left[C^{(O F F)}-C^{(O N)}\right]=\frac{1}{2}\left(\frac{I_{0}^{(O F F)}-I_{1}^{(O F F)}}{I_{0}^{(O F F)}+I_{1}^{(O F F)}}-\frac{I_{0}^{(O N)}-I_{1}^{(O N)}}{I_{0}^{(O N)}+I_{1}^{(O N)}}\right)
$$

If the intensity switches completely from zeroth to first order the $(F O M)$ value is equal to 1 , if from first to zeroth order, -1. If a beam deflector doesn't switch intensity between the orders at all, the FOM number vanishes, even if there is a change in the total intensity. Thus, the absolute value of the FOM number shows the quality of the beam deflection. Ideally, we should target to achieve $F O M=1$ or close to it.

For our fabricated beam deflector, we achieve $F O M=0.48$. The numerical simulations showed in Figure 4 predicted FOM of about 0.45 demonstrating a very good match between the theoretical results and experimental data.

In summary, we have demonstrated how to employ liquid crystals for achieving func- 
tional tunability of optical metasurface devices. In particular, employing the concept of tunable resonant Huygens' metasurfaces, we have studied theoretically and demonstrated experimentally a switchable beam deflection metadevice operating in the visible spectrum. By controlling temperature of the metasurface, we have been able to control the amount of the optical power propagating straight (zero order) or deflected at $12^{\circ}$ to the normal (first order). We notice that the thickness of such a metadevice is only $5 \mu m$, with the minimal thickness of a liquid crystal layer being limited only by the near field of silicon nanoantennas that is significantly smaller than $1 \mu \mathrm{m}$. Thus, switchable beam steering metadevices of the thickness smaller than $1 \mu m$ can be realized in practice without compromising their efficiency. WQe believe our results open new opportunities for dynamically reconfigurable functional metadevices including tunable lenses and dynamic metasurface displays.

\section{Methods}

Fabrication of samples. To fabricate the samples we deposited a layer of amorphous silicon film with a controlled thicknesses of $130 \mathrm{~nm}$ on a quartz substrate using the inductively coupled plasma chemical vapor deposition technique (Plasmalab System 380, Oxford Instruments). Thereafter, we patterned hydrogen silsesquioxane (HSQ, Dow Corning, XR1541-006) arrays on the silicon film using the electron beam lithography (Elionix, $100 \mathrm{kV}$ ) method. The silicon metasurface is finally obtained through silicon film etching using an inductively coupled plasma etcher (Plasmalab System 100, Oxford) with $\mathrm{Cl}_{2}$ gas.

Experimental setup. To perform measurements of the beam deflection we used an experimental setup shown in the Figure S2. As a light source we used a tunable femtosecond laser with pulse duration of $200 \mathrm{fs}$ and repetition rate of $80 \mathrm{MHz}$. The spectral full-width at half-maximum of the laser line is $4.4 \mathrm{~nm}$. The intensity of the laser was kept at a very low value to avoid nonlinear effects associated with high laser power. The lens after the laser focused the beam onto the cell surface to match the diameter of light beam and the 
dimensions of the silicon-disk array $\left(75 \times 75 \mu \mathrm{m}^{2}\right)$. Microscope objective with magnification $\times 100$ collects the transmitted light and magnifies the image which we are able to capture with a camera and a tube lens installed at the focal distance in front of the camera. By introducing and removing another lens behind the objective we are able to image the back focal plane of the objective and switch between the real and $k$-space.

\section{Acknowledgement}

We acknowledge a financial support from the Australian Research Council through Discovery Projects, the CUDOS research programs, and the use of the Australian National Fabrication Facility (ANFF) at the ACT Node. DSI co-authors were supported in Singapore by A*STAR SERC Pharos program (Grant 15273 00025) and National Research Foundation Investigatorship Award (Grant NRF-NRFI2017-01).

\section{Supporting Information Available}

The supporting information includes details of the sample design and fabrication as well as an experimental setup for experimental measurements. This material is available free of charge via the Internet at http://pubs.acs.org/.

\section{References}

(1) Kuznetsov, A. I.; Miroshnichenko, A. E.; Brongersma, M. L.; Kivshar, Y. S.; Luk'yanchuk, B. Optically resonant dielectric nanostructures. Science 2016, 354, aag2472.

(2) Zhu, A. Y.; Kuznetsov, A. I.; Lukyanchuk, B.; Engheta, N.; Genevet, P. Traditional and emerging materials for optical metasurfaces. Nanophotonics 2017, 6, 452-471. 
(3) Lalanne, P.; Chavel, P. Metalenses at visible wavelengths: past, present, perspectives. Laser Photonics Rev. 2017, 11, 1600295.

(4) Arbabi, A.; Horie, Y.; Ball, A. J.; Bagheri, M.; Faraon, A. Subwavelength-thick lenses with high numerical apertures and large efficiency based on high-contrast transmitarrays. Nat. Commun. 2015, 6, 7069.

(5) Khorasaninejad, M.; Chen, W. T.; Devlin, R. C.; Oh, J.; Zhu, A. Y.; Capasso, F. Metalenses at visible wavelengths: Diffraction-limited focusing and subwavelength resolution imaging. Science 2016, 352, 1190-1194.

(6) Paniagua-Dominguez, R.; Yu, Y. F.; Khaidarov, E.; Bakker, R. M.; Liang, X.; Fu, Y. H.; Kuznetsov, A. I. A Metalens with Near-Unity Numerical Aperture. arXiv preprint arXiv:1705.00895 2017,

(7) Chen, B. H.; Wu, P. C.; Su, V.-C.; Lai, Y.-C.; Chu, C. H.; Lee, I. C.; Chen, J.-W.; Chen, Y. H.; Lan, Y.-C.; Kuan, C.-H.; Tsai, D. P. GaN Metalens for Pixel-Level FullColor Routing at Visible Light. Nano Lett. 2017, 17, 6345-6352.

(8) Lin, D.; Fan, P.; Hasman, E.; Brongersma, M. L. Dielectric gradient metasurface optical elements. Science 2014, 345, 298-302.

(9) Yu, Y. F.; Zhu, A. Y.; Paniagua-Domínguez, R.; Fu, Y. H.; Luk'yanchuk, B.; Kuznetsov, A. I. High-transmission dielectric metasurface with $2 \pi$ phase control at visible wavelengths. Laser Photon. Rev. 2015, 9, 412-418.

(10) Chong, K. E.; Staude, I.; James, A.; Dominguez, J.; Liu, S.; Campione, S.; Subramania, G. S.; Luk, T. S.; Decker, M.; Neshev, D. N.; Brener, I.; Kivshar, Y. S. PolarizationIndependent Silicon Metadevices for Efficient Optical Wavefront Control. Nano Lett. 2015, 15, 5369-5374. 
(11) Lin, D.; Melli, M.; Poliakov, E.; Hilaire, P. S.; Dhuey, S.; Peroz, C.; Cabrini, S.; Brongersma, M.; Klug, M. Optical metasurfaces for high angle steering at visible wavelengths. Sci. Rep. 2017, r, 2286.

(12) Khaidarov, E.; Hao, H.; Paniagua-Dominguez, R.; Yu, Y.; Fu, Y. H.; Valuckas, V.; Yap, S., Lee Koon; Toh, Y. T.; Ng, J., Siu Kit; Kuznetsov, A. I. Asymmetric nanoantennas for ultra-high angle broadband visible light bending. Nano Lett. 2017, 17, 62676272.

(13) Chalabi, H.; Ra'di, Y.; Sounas, D.; Alù, A. Efficient anomalous reflection through near-field interactions in metasurfaces. Phys. Rev. B 2017, 96, 075432.

(14) Sell, D.; Yang, J.; Doshay, S.; Yang, R.; Fan, J. A. Large-Angle, Multifunctional Metagratings Based on Freeform Multimode Geometries. Nano Lett. 2017, 17, 3752-3757.

(15) Arbabi, A.; Horie, Y.; Bagheri, M.; Faraon, A. Dielectric metasurfaces for complete control of phase and polarization with subwavelength spatial resolution and high transmission. Nat. Nanotechnol. 2015, 10, 937-943.

(16) Wang, L.; Kruk, S.; Tang, H.; Li, T.; Kravchenko, I.; Neshev, D. N.; Kivshar, Y. S. Grayscale transparent metasurface holograms. Optica 2016, 3, 1504-1505.

(17) Chong, K. E.; Wang, L.; Staude, I.; James, A. R.; Dominguez, J.; Liu, S.; Subramania, G. S.; Decker, M.; Neshev, D. N.; Brener, I.; Kivshar, Y. S. Efficient PolarizationInsensitive Complex Wavefront Control Using Huygens' Metasurfaces Based on Dielectric Resonant Meta-atoms. ACS Photonics 2016, 3, 514-519.

(18) Fu, Y. H.; Kuznetsov, A. I.; Miroshnichenko, A. E.; Yu, Y. F.; Luk'yanchuk, B. Directional visible light scattering by silicon nanoparticles. Nat. Commun. 2013, 4, 1527.

(19) Person, S.; Jain, M.; Lapin, Z.; Saenz, J. J.; Wicks, G.; Novotny, L. Demonstration of zero optical backscattering from single nanoparticles. Nano Lett. 2013, 13, 1806-1809. 
(20) Paniagua-Domínguez, R.; Yu, Y. F.; Miroshnichenko, A. E.; Krivitsky, L. A.; Fu, Y. H.; Valuckas, V.; Gonzaga, L.; Toh, Y. T.; Kay, A. Y. S.; Lukyanchuk, B.; Kuznetsov, A. I. Generalized Brewster effect in dielectric metasurfaces. Nat. Commun. 2016, 7, 10362.

(21) Decker, M.; Staude, I.; Falkner, M.; Dominguez, J.; Neshev, D. N.; Brener, I.; Pertsch, T.; Kivshar, Y. S. High-Efficiency Dielectric Huygens' Surfaces. Adv. Opt. Mater. 2015, 3, 813-820.

(22) Staude, I.; Miroshnichenko, A. E.; Decker, M.; Fofang, N. T.; Liu, S.; Gonzales, E.; Dominguez, J.; Luk, T. S.; Neshev, D. N.; Brener, I.; Kivshar, Y. Tailoring Directional Scattering through Magnetic and Electric Resonances in Subwavelength Silicon Nanodisks. ACS Nano 2013, 7, 7824-7832.

(23) Campione, S.; Basilio, L. I.; Warne, L. K.; Sinclair, M. B. Tailoring dielectric resonator geometries for directional scattering and Huygens' metasurfaces. Opt. Express 2015, 23, 2293-2307.

(24) Zheludev, N. I.; Kivshar, Y. S. From metamaterials to metadevices. Nat. Mater. 2012, 11, 917.

(25) Huang, Y.-W.; Lee, H. W. H.; Sokhoyan, R.; Pala, R. A.; Thyagarajan, K.; Han, S.; Tsai, D. P.; Atwater, H. A. Gate-tunable conducting oxide metasurfaces. Nano Lett. 2016, 16, 5319-5325.

(26) Kamali, S. M.; Arbabi, E.; Arbabi, A.; Horie, Y.; Faraon, A. Highly tunable elastic dielectric metasurface lenses. Laser Photonics Rev. 2016, 10, 1002-1008.

(27) She, A.; Zhang, S.; Shian, S.; Clarke, D. R.; Capasso, F. Large Area Electrically Tunable Lenses Based on Metasurfaces and Dielectric Elastomer Actuators. arXiv preprint arXiv:1708.01972 2017, 
(28) Iyer, P. P.; Pendharkar, M.; Schuller, J. A. Electrically Reconfigurable Metasurfaces Using Heterojunction Resonators. Adv. Opt. Mater. 2016, 4, 1582-1588.

(29) Lewi, T.; Evans, H. A.; Butakov, N. A.; Schuller, J. A. Ultrawide Thermo-optic Tuning of PbTe Meta-Atoms. Nano Lett. 2017, 17, 3940-3945.

(30) Rahmani, M.; Xu, L.; Miroshnichenko, A. E.; Komar, A.; Camacho-Morales, R.; Chen, H.; Zárate, Y.; Kruk, S.; Zhang, G.; Neshev, D. N.; Kivshar, Y. S. Reversible Thermal Tuning of All-Dielectric Metasurfaces. Adv. Funct. Mater. 2017, 1700580.

(31) Karvounis, A.; Gholipour, B.; MacDonald, K. F.; Zheludev, N. I. All-dielectric phasechange reconfigurable metasurface. Appl. Phys. Lett. 2016, 109, 051103.

(32) Wang, Q.; Rogers, E. T. F.; Gholipour, B.; Wang, C.-M.; Yuan, G.; Teng, J.; Zheludev, N. I. Optically reconfigurable metasurfaces and photonic devices based on phase change materials. Nat. Photonics 2016, 10, 60-65.

(33) Sun, T.; Kim, J.; Yuk, J. M.; Zettl, A.; Wang, F.; Chang-Hasnain, C. Surface-normal electro-optic spatial light modulator using graphene integrated on a high-contrast grating resonator. Opt. Express 2016, 24, 26035-26043.

(34) Sautter, J.; Staude, I.; Decker, M.; Rusak, E.; Neshev, D. N.; Brener, I.; Kivshar, Y. S. Active Tuning of All-Dielectric Metasurfaces. ACS Nano 2015, 9, 4308-4315.

(35) Komar, A.; Fang, Z.; Bohn, J.; Sautter, J.; Decker, M.; Miroshnichenko, A.; Pertsch, T.; Brener, I.; Kivshar, Y. S.; Staude, I.; Neshev, D. N. Electrically tunable all-dielectric optical metasurfaces based on liquid crystals. Appl. Phys. Lett. 2017, 110, 071109.

(36) Parry, M.; Komar, A.; Hopkins, B.; Campione, S.; Liu, S.; Miroshnichenko, A. E.; Nogan, J.; Sinclair, M. B.; Brener, I.; Neshev, D. N. Active tuning of high-Q dielectric metasurfaces. Appl. Phys. Lett. 2017, 111, 053102. 
(37) Li, J.; Wu, S.-T.; Brugioni, S.; Meucci, R.; Faetti, S. Infrared refractive indices of liquid crystals. J. Appl. Phys. 2005, 97, 073501. 